\title{
Weighting GPS Dual Frequency Observations: Bearing the Cross of Cross-Correlation
}

\author{
P.J.G. Teunissen, N.F. Jonkman, C.C.J.M. Tiberius \\ Department of Mathematical Geodesy and Positioning \\ Delft University of Technology \\ The Netherlands \\ e-mail:mgp@geo.tudelft.nl
}

\begin{abstract}
A proper choice of the observation weight matrix is of importance for both adjusting and testing GPS data. Our understanding of the noise characteristics of GPS observations, on which the weight matrix should be based, is however still underdeveloped. This makes it difficult to draw up an appropriate weight matrix. The first and foremost purpose of this contribution is therefore to draw attention to the need to improve upon our rudimentary knowledge of the GPS stochastic model. To this end, results will be presented of a relatively simple casestudy in which the possible presence of cross-correlation between observables is considered. With these results we hope to spur further discussion and research on this important topic.
\end{abstract}

\section{INTRODUCTION}

GPS data are usually processed with algorithms based on the least-squares principle. In order to apply the least-squares principle one needs to specify both the observation equations and the observation weights. The observation equations link the GPS observables, like pseudo ranges and carrier phases, to the unknown parameters, such as, for example, baseline coordinates, carrier phase ambiguities and atmospheric delays. The observation weights, which are collected in a weight matrix, allow one to specify by how much the individual observations should contribute to the overall solution. For instance, it is sensible to give lower weights to the noisier observations and higher weights to the less noisy observations. The choice of weights is optimal when the weight matrix equals the inverse of the variance-covariance (vc-) matrix of the observations. In that case the balance between the relative weights is such that the best possible precision is obtained in the computed solution.

The GPS observation equations are (sufficiently) known and well documented. However, the same can not be said of the vc-matrix of the GPS observations. In the many GPS-textbooks available, one will usually find only a few comments, if any, on the vc-matrix of the GPS observations. Also advertisement or data sheets for GPS receivers are usually vague in their specifications of the precision characteristics of the data outputted by the receiver. Due to this lack of information in the public domain, most of us are probably inclined to start with the simplest weight matrix possible, a scaled unit matrix for instance per observation type (pseudo ranges and carrier phases). Such a choice may however be an oversimplification that fails to do justice to the more complicated noise characteristics of the data.

A proper choice of the vc-matrix is of relevance for all subsequent stages of data processing. The least-squares solution for instance, will loose its property of 'minimum variance' when a misspecified vc-matrix is used. In addition, the detection power of the statistical tests, employed for model validation and quality control (e.g. outliers and cycle-slips), will become smaller when the noise characteristics are not properly taken into account. And finally, the a posteriori quality description of the computed results will also be affected when mispecified or oversimplified vc-matrices are used.

At present research into the stochastic model of GPS observables is still in its infancy. Only a few studies have been reported in the literature. Examples are [Euler and Goad, 1991], [Jin and de Jong, 1996], [Gerdan, 1995] and [Gianniou, 1996], who studied the elevation dependence of the observation variances, and [Jonkman, 1998] and [Tiberius, 1998], who considered time-correlation and cross-correlation of the pseudo ranges and carrier phases as well.

A systematic study of the stochastic model is of course far from trivial. Not only do the noise characteristics depend on the mechanization of the measurement process, and therefore on the make and type of the receiver used, but the residual terms which are not captured by the observation equations, such as environmental effects, will also have their influence. Despite these difficulties though, we believe that the time has come to put more effort into the stochastic 
model and to try to come up with a more qualitative description of the noise characteristics. This contribution is therefore foremost a plea for having more research done in this area. By directing attention to the noise characteristics and sharing the knowledge so obtained, one will hopefully be able to link the stochastic model more firmly to one's data. To underline the need for this type of research, this contribution presents the results of a relatively simple casestudy in which the possible presence of crosscorrelation between the observables is the topic of discussion. It illustrates that in some cases the use of a scaled unit matrix or even a diagonal matrix as vcmatrix will result in an unnecessary oversimplification of the noise characteristics. Nondiagonal vc-matrices for the pseudo ranges and carrier phases are suggested instead.

\section{PSEUDORANGE NOISE}

The choice of the weight matrix or its inverse, the vcmatrix, should reflect the noise properties of the observations. A first impression of the noise characteristics can be obtained from appropriately constructed time series of the data. For that purpose we consider time series of

$$
\begin{aligned}
& y_{1}(i)=p_{1}(i)-\phi_{1}(i)+\lambda_{1} a_{1} \\
& y_{2}(i)=p_{2}(i)-\phi_{2}(i)+\lambda_{2} a_{2}
\end{aligned}
$$

where $p_{1}(i), p_{2}(i)$ are the double differenced (DD) pseudo range (code) observables on L1 resp. L2 at epoch $i ; \phi_{1}(i), \phi_{2}(i)$ are the DD carrier phase observables on L1 resp. L2 at epoch $i$, expressed in units of range rather than cycles; $a_{1}, a_{2}$ are the timeinvariant integer DD ambiguities; and $\lambda_{1}, \lambda_{2}$ are the wavelengths of L1 resp. L2.

The reason for using double differenced data is that it allows the elimination of the unknown receiver and satellite clock errors. The DD ambiguities are in addition known to be integers. In fact, in our analysis these two ambiguities were fixed. The integer values were determined reliably using a sufficiently long time span of data. The data were measured at a 1 second sampling rate on a zero-baseline, so as to eliminate to a sufficient extent the potential contributions of the ionospheric delays (and environmental effects). As a consequence, the noise characteristics of $y_{1}(i), y_{2}(i)$, which have zero mean, are predominantly those of $p_{1}(i), p_{2}(i)$. The noise contributions of the phase data are namely so small (about a factor of one hundred smaller), that they can safely be neglected.

Figure 1 shows an example of three time series for the satellite-pair 01-25. The first is a time series of $y_{1}(i)$, the second of $y_{2}(i)$ and the third is the time series of the difference $y_{2}(i)-y_{1}(i)$. The first two time series give an impression of the pseudo range noise on L1 and L2, and thus of their DD variances $\sigma_{p 1}^{2}$ and $\sigma_{p 2}^{2}$. The third time series
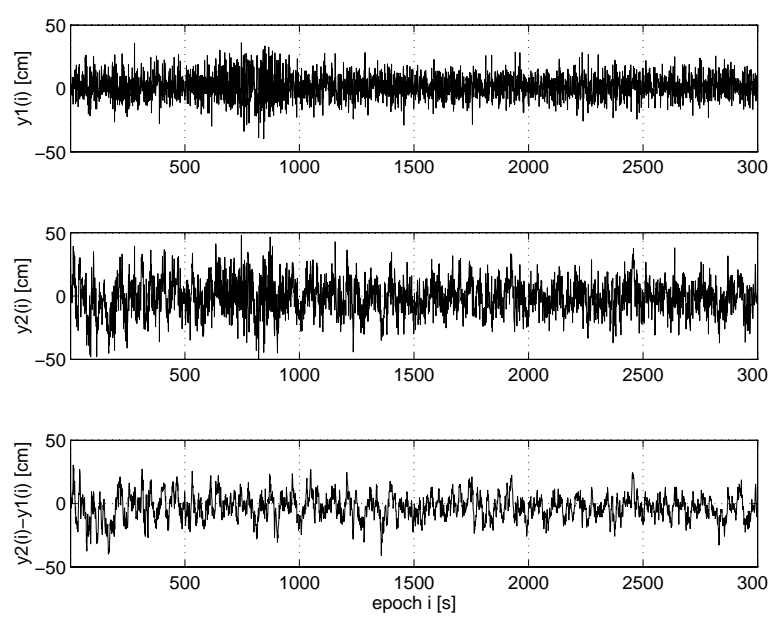

Figure 1: Time series of $y_{1}(i)$ (top), $y_{2}(i)$ (middle) and their difference $y_{2}(i)-y_{1}(i)$ (bottom).

gives an impression of the noise of the difference between the two pseudo ranges. Using the error propagation law, the variance of the pseudo range difference $\Delta p=p_{2}-p_{1}$ can be expressed in the pseudo range variances as

$\sigma_{\Delta p}^{2}=\sigma_{p_{2}}^{2}-2 \sigma_{p_{2} p_{1}}+\sigma_{p_{1}}^{2}$

where $\sigma_{p 2 p l}$ denotes the covariance between $p_{2}$ and $p_{1}$. When specifying the weight matrix or vc-matrix, one will usually be inclined to set $\sigma_{p 2 p 1}$ equal to zero, thereby assuming that the receiver outputted dual frequency pseudo range data are independent and thus uncorrelated. But if that is the case, one would expect the noise in the pseudo range difference to be larger than the noise in either one of the two pseudo ranges. A closer look at figure 1 shows however that this is not the case. The noise of $\Delta p$ is in fact somewhat smaller than that of $p_{2}$. This indicates the presence of positive correlation between the two pseudo ranges.

In order to quantify the pseudo range noise characteristics of this example, the data were used to estimate the pseudo range vc-matrix. The estimated matrix reads

$C_{p}=\left[\begin{array}{cc}91.6 & 90.4 \\ 90.4 & 185.4\end{array}\right]$

where the matrix entries are expressed in $\mathrm{cm}^{2}$. The matrix is clearly not diagonal. The pseudo range variance of L2 is about twice as large as that of L1 and the correlation differs significantly from zero. The correlation coefficient equals $\rho=0.69$. This shows that the vc-matrix of the pseudo range data in this example is not a scaled unit matrix nor a diagonal matrix.

\section{CROSS-CORRELATION}

From the above example it will be clear that the use of a diagonal matrix as vc-matrix would be an 
oversimplification of the L1/L2 noise characteristics. The receiver outputted L1/L2 data are simply not stochastically independent. The stochastic properties of outputted GPS data depend to a large extent on how the measurement process is mechanized inside the receiver. For instance, to circumvent the $\mathrm{P}$-code encryption (AntiSpoofing) implemented by the Department of Defence (DoD), so-called codeless and semi-codeless techniques were developed. An overview of these measurement techniques can be found in e.g. [Ashjaee and Lorenz, 1992], [Dierendonck, 1995], [Langley, 1996] and [Hofmann-Wellenhof et al., 1997]. One of these technique is cross-correlation. It is based on the fact that both the L1 and L2 carrier are modulated (coherently) with the same P- (or Y-) code. This allows the receiver to measure the difference of the L1 and L2 pseudo ranges and the difference of the L1 and L2 carrier phases without knowledge of the actual (secret) Y-code when AS is on.

As a measurement technique, cross-correlation has its origin in interferometry. In the early eighties, the Jet Propulsion Laboratory (JPL) developed the SERIES (Satellite Emission Range Inferred Earth Surveying)receiver, see [MacDoran, 1979] and [MacDoran et al., 1985]. The signal group delay measured between two sites by correlation can be considered as (differential) codeless pseudo ranging. As such this receiver is the ancestor of the cross-correlation engines of the nineties. Geodetic receivers that, to our knowledge, employ cross-correlation to provide dual frequency code and phase observations are the TurboRogue SNR-8000 [Meehan et al., 1992], the 4000 series (SSE/SSi) of Trimble [Trimble, 1994] and the Sercel 5002/6002 [Barboux, 1997].

\subsection{Pseudo range cross-correlation}

The cross-correlation technique was implemented in the measurement process that formed the basis of the time series presented in the previous section. We will therefore try to come up with a simple, but hopefully effective description of the vc-matrix of such reconstructed $\mathrm{L} 1 / \mathrm{L} 2$ data.

In case of the pseudo range data one can argue that not $p_{1}$ and $p_{2}$ are the independent observables, but rather $p_{1}$ and the difference $\Delta p=p_{2}-p_{1}$. The outputted L1 and L2 pseudo ranges are then reconstructed as

$$
p_{1}=p_{1} \quad \text { and } \quad p_{2}=p_{1}+\Delta p
$$

If we now apply the error propagation law and assume $p_{l}$ and $\Delta p$ to be uncorrelated, the resulting vc-matrix becomes

$$
C_{p}=\left[\begin{array}{cc}
\sigma_{p_{1}}^{2} & \sigma_{p_{1}}^{2} \\
\sigma_{p_{1}}^{2} & \sigma_{p_{1}}^{2}+\sigma_{\Delta p}^{2}
\end{array}\right]
$$

This matrix is clearly nondiagonal. It is nondiagonal as a consequence of the way in which the L2 pseudo range is reconstructed from $p_{1}$ and $\Delta p$. Note how well this matrix fits the previous empirical estimate of the vcmatrix.

To infer the significance of the correlation between $p_{l}$ and $p_{2}$, we consider the correlation coefficient. It reads

$$
\rho_{p}=\frac{1}{\sqrt{1+\sigma_{\Delta p}^{2} / \sigma_{p_{1}}^{2}}}
$$

This shows that the correlation coefficient equals 0.71 for a variance ratio of one and that it gets smaller the larger the variance ratio becomes. Thus one is only allowed to approximate the vc-matrix $C_{p}$ by a diagonal matrix when the variance of $\Delta p$ is much larger than that of $p_{l}$. But it will then not be a scaled unit matrix, since $\sigma_{p_{2}}>\sigma_{p_{1}}$.

\subsection{Carrier phase cross-correlation}

For the carrier phase data one can argue, somewhat analogous to the pseudo range case, that not $\phi_{1}$ and $\phi_{2}$ are the independent observables, but rather $\phi_{1}$ and the difference of the two phases. This difference however, is now taken in the domain of cycles instead of in the range domain. In essence this means that the second independent observable is not $\phi_{2}$ but rather the widelane carrier phase observable

$\phi_{w}=\lambda_{w}\left(\frac{\phi_{2}}{\lambda_{2}}-\frac{\phi_{1}}{\lambda_{1}}\right)$

with the widelane wavelength $\lambda_{w}=\left(1 / \lambda_{1}-1 / \lambda_{2}\right)^{-1}$. Hence the outputted L1 and L2 carrier phases, when expressed in units of range rather than cycles, are reconstructed as

$$
\phi_{1}=\phi_{1} \quad \text { and } \quad \phi_{2}=\lambda_{2}\left(\frac{\phi_{1}}{\lambda_{1}}+\frac{\phi_{w}}{\lambda_{w}}\right)
$$

If we now apply the error propagation law and assume $\phi_{1}$ and $\phi_{w}$ to be uncorrelated, the resulting vc-matrix becomes

$$
C_{\phi}=\left[\begin{array}{cc}
\sigma_{\phi_{1}}^{2} & \left(\lambda_{2} / \lambda_{1}\right) \sigma_{\phi_{1}}^{2} \\
\left(\lambda_{2} / \lambda_{1}\right) \sigma_{\phi_{1}}^{2} & \left(\lambda_{2} / \lambda_{1}\right)^{2} \sigma_{\phi_{1}}^{2}+\left(\lambda_{2} / \lambda_{w}\right)^{2} \sigma_{\phi_{w}}^{2}
\end{array}\right]
$$

Again this matrix is nondiagonal. The correlation coefficient of the two carrier phases reads

$$
\rho_{\phi}=\frac{1}{\sqrt{1+\left(\sigma_{\phi_{w}} / \lambda_{w}\right)^{2} /\left(\sigma_{\phi_{1}} / \lambda_{1}\right)^{2}}}
$$

This shows that the correlation coefficient gets smaller the larger the widelane phase variance gets. Thus for a large enough value one may approximate the vc-matrix by a diagonal matrix. The two diagonal entries will then differ though, since $\sigma_{\phi_{2}}>\sigma_{\phi_{1}}$.

\section{CONSEQUENCES}

Dual-frequency receivers may output their data directly on the L1/L2 frequencies, or the data are made 
available via the Rinex format [Gurtner, 1994]. Hence with two receivers, a user will have at his disposal the DD phase and pseudo range observables $\phi_{1}, \phi_{2}, p_{1}, p_{2}$. The observations can be linked to the unknown parameters according to the following DD observation equations

$$
\begin{aligned}
& \phi_{1}(i)=\rho(i)+T(i)-\mu_{1} I(i)+\lambda_{1} a_{1}+n_{\phi_{1}}(i) \\
& \phi_{2}(i)=\rho(i)+T(i)-\mu_{2} I(i)+\lambda_{2} a_{2}+n_{\phi_{2}}(i) \\
& p_{1}(i)=\rho(i)+T(i)+\mu_{1} I(i)+n_{p_{1}}(i) \\
& p_{2}(i)=\rho(i)+T(i)+\mu_{2} I(i)+n_{p_{2}}(i)
\end{aligned}
$$

where $\rho(i)$ equals the DD range at epoch $i, T(i)$ and $I(i)$ are the tropospheric resp. ionospheric delays at epoch $i$, $\mu_{1}$ and $\mu_{2}$ are the wavelength ratios $\mu_{1}=\lambda_{1} / \lambda_{2}$ and $\mu_{2}=\lambda_{2} / \lambda_{1}$, and $n_{\phi 1}(i), n_{\phi 2}(i), n_{p 1}(i), n_{p 2}(i)$ denote the respective noise terms.

The observables of Eq.(4.1) are not stochastically independent if the cross-correlation technique is used. This implies that the user has two options available if he wants to apply a proper weighting of his data. Either he uses the receiver outputted data directly, in which case he will have to work with the nondiagonal vcmatrices $C_{p}$ and $C_{\phi}$, or he back-transforms his data first to the original uncorrelated observables $\phi_{1}, \phi_{w}, p_{1}, \Delta p$, in which case he can work with a diagonal vc-matrix again. In the latter case, the observation equations will have to be transformed as well of course. Both approaches will give identical results, provided the correct vc-matrices are used. Different, and in fact less precise, results will be obtained when the first approach is used while still assuming that the data are correlation-free.

\subsection{Effect on the least-squares ambiguities}

In order to illustrate the effect of using diagonal vcmatrices instead of the nondiagonal ones, $C_{p}$ and $C_{\phi}$, we will consider the least-squares ambiguity estimates and their formal and empirical precision. We will use the observation equations of Eq.(4.1) and assume that the baseline is sufficiently short so as to neglect the ionospheric delays, i.e. $I(i)=0$. This is the typical assumption for most surveying applications. We also keep the observation equations parametrized in terms of the DD ranges. That is, they are not parametrized further in terms of the baseline coordinates. For ambiguity resolution this is the simplest model one can think of. The model is linear and no special precautions need to be taken for the tropospheric delays, since these delays are automatically lumped with the corresponding DD ranges. As a consequence, the solution for the ambiguities is bias-free as far as the contribution of the troposphere is concerned.

Based on these observation equations for a single epoch of data, the least-squares solution for the DD ambiguities, when using scaled unit matrices as vcmatrices for respectively the pseudo ranges and carrier phases, reads

$$
\begin{aligned}
& \hat{a}_{1}(i)=\frac{1}{\lambda_{1}}\left[\phi_{1}(i)-\frac{1}{2}\left(p_{1}(i)+p_{2}(i)\right)\right] \\
& \hat{a}_{2}(i)=\frac{1}{\lambda_{2}}\left[\phi_{2}(i)-\frac{1}{2}\left(p_{1}(i)+p_{2}(i)\right)\right]
\end{aligned}
$$

Hence, the least-squares ambiguities are equal to the differences between the carrier phases, expressed in units of range, and the average of the pseudo ranges.

Would we use the same observation equations, but now with the nondiagonal vc-matrices $C_{p}$ and $C_{\phi}$, rather than with diagonal vc-matrices, then the least-squares solution becomes

$$
\begin{aligned}
& \hat{a}_{1}^{\prime \prime}(i)=\frac{1}{\lambda_{1}}\left[\phi_{1}(i)-p_{1}(i)\right] \\
& \hat{a}_{2}^{\prime \prime}(i)=\frac{1}{\lambda_{2}}\left[\phi_{2}(i)-p_{1}(i)\right]
\end{aligned}
$$

When we compare this solution with the previous one, we immediately notice a distinct difference. In the first solution, Eq.(4.2), it is the average of the two pseudo ranges that contributes to the solution, whereas in Eq.(4.3) it is only the L1 pseudo range that contributes. The first solution depends therefore on all four types of observables, whereas the second solution depends only on three.

\subsection{Effect on the precision of the L1-ambiguity}

Not using one of the available observations, while still claiming that a more precise solution is obtained, may seem strange at first sight. It is a direct consequence however of using the nondiagonal vc-matrices $C_{p}$ and $C_{\phi}$. It is solely due to their structure that the L2 pseudo ranges get eliminated from the solution.

To make these findings more plausible, we will first consider the formal precision of the single epoch L1 ambiguity estimates. If we assume the L1 and L2 pseudo ranges to be uncorrelated and of equal variance $\sigma_{p}^{2}=\sigma_{p 1}^{2}=\sigma_{p 2}^{2}$, application of the error propagation law to Eq.(4.2) yields

$$
\sigma_{\hat{a} 1}^{2}=\frac{1}{\lambda_{1}^{2}}\left(\sigma_{\phi 1}^{2}+\frac{1}{2} \sigma_{p}^{2}\right)
$$

If instead the L1 and L2 pseudo ranges are assumed to be correlated according the non-diagonal vc-matrix $C_{p}$, application of the error propagation law to Eq.(4.3) yields

$$
\sigma_{\hat{a} 1^{\prime \prime}}^{2}=\frac{1}{\lambda_{1}^{2}}\left(\sigma_{\phi 1}^{2}+\sigma_{p}^{2}\right)
$$

On comparing these equations, it is perfectly clear that the use of both the L1 and L2 pseudo ranges results in more precise ambiguity estimates provided that the L1 and L2 pseudo ranges are uncorrelated.

If however, we assume the L1 and L2 pseudo ranges uncorrelated and therefore use the estimation formula Eq.(4.2), while the pseudo ranges are actually correlated according to the vc-matrix $C_{p}$, we will obtain 
less precise ambiguity estimates. To illustrate this fact, the error propagation law is applied to Eq.(4.2) with the vc-matrix $C_{p}$. This yields

$$
\sigma_{\hat{a} 1}^{2}=\frac{1}{\lambda_{1}^{2}}\left(\sigma_{\phi 1}^{2}+\sigma_{p}^{2}+\frac{1}{4} \sigma_{\Delta p}^{2}\right)
$$

It appears from this equation that under this assumption the inclusion of the L2 pseudo ranges in the solution does not improve the precision of the ambiguity estimates. On the contrary, the precision of the ambiguities estimated with the L2 pseudo ranges is even worse than the precision of the ambiguities estimated without the L2 pseudo ranges.
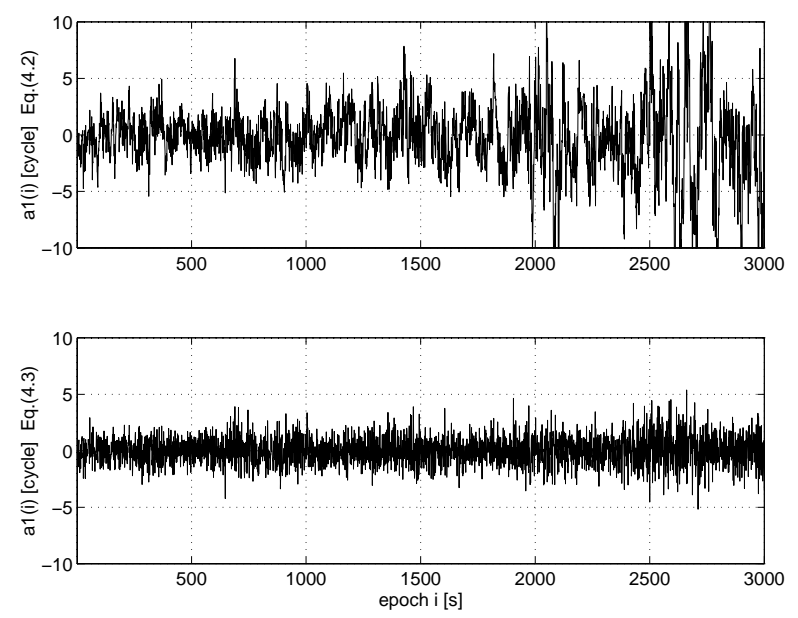

Figure 2: Time series of $a_{1}(i)$ estimated according to Eq.(4.2) (top) and according to Eq.(4.3) (bottom).

In practice the improvement in precision of the ambiguity estimates resulting from the elimination of the L2 pseudo ranges in the solution can take on a rather dramatic form. Consider to that end figure 2 . This figure contains time series of L1 ambiguities estimated from zero baseline data according to Eq.(4.2) (top) and according Eq.(4.3) (bottom). On comparing the time series in the figure it is clear that the noise in the ambiguities estimated with the L2 pseudo ranges is indeed considerably larger than the noise in the ambiguities estimated without the L2 pseudo ranges. In addition it appears from figure 2 (top) that the noise in the ambiguities estimated with the L2 pseudo ranges increases notably towards the end of the observation interval. This in fact is another consequence of the cross-correlation technique. Application of crosscorrelation results namely in a smaller signal-to-noiseratio (SNR) as compared with code correlation, see e.g. [Ashjaee and Lorenz, 1992]. Hence, the noise in the cross-correlation derived pseudo range (and carrier phase) differences is expected to exhibit a pronounced dependence on the satellite elevation. And as one satellite of the pair considered for figure 2 sets during the observation span, this may well explain the progressive increase of the noise in the ambiguities estimated according to Eq.(4.2).

\subsection{Effect on the precision of the L1-L2 ambiguity pair}

In the previous section we considered the formal precision of the single epoch L1 ambiguity estimates. However, as we simultaneously estimate both L1 and L2 ambiguities, it is more opportune to consider the formal precision of the single epoch dual frequency ambiguity estimates. To that end, the error propagation law is applied to Eq.(4.2) under the assumption that the observables are uncorrelated and to Eq.(4.3) under the assumption that the observables are correlated according to the vc-matrices $C_{p}$ and $C_{\phi}$. The resulting formal ambiguity vc-matrices will be denoted by $C_{a}$ and $C_{a}^{\prime \prime}$.

It is of course not enough to restrict attention to the formal precision alone. After all, if we want to study the noise characteristics of the data, we should let the data speak for themselves. In addition to the formal precision, we therefore also need its empirical counterpart. The empirical precision of the ambiguities is described by their empirically determined vc-matrix. For the ambiguities estimated according to Eq.(4.2), this matrix is computed as

$$
\hat{C}_{a}=\frac{1}{k-1} \sum_{i=1}^{k}\left[\begin{array}{l}
\hat{a}_{1}(i)-\bar{a}_{1} \\
\hat{a}_{2}(i)-\bar{a}_{2}
\end{array}\right]\left[\begin{array}{l}
\hat{a}_{1}(i)-\bar{a}_{1} \\
\hat{a}_{2}(i)-\bar{a}_{2}
\end{array}\right]^{T}
$$

where $k$ denotes the number of samples - 3000 in our case - and $\bar{a}_{1}$ and $\bar{a}_{2}$ denote the ambiguity sample means. For the ambiguities estimated according to Eq.(4.3), the empirical vc-matrix is computed in a similar way and denoted as $\hat{C}_{a}^{\prime \prime}$.

Both the formal and empirical dual frequency ambiguity precision will be shown by means of $95 \%$ standard ellipses. However, as the L1 and L2 ambiguity estimates are strongly correlated, the ellipses corresponding to the vc-matrices $C_{a}$ and $C_{a}^{\prime \prime}$ and to the vc-matrices $\hat{C}_{a}$ and $\hat{C}_{a}^{\prime \prime}$ are extremely elongated. In order to allow a better interpretation of the precision results, a decorrelating LAMBDA transformation, see [Teunissen, 1995], was applied to these matrices. The standard ellipses depicted in this section correspond consequently to the transformed vc-matrices $C_{z}$ and $C_{z}^{\prime \prime}$, and $\hat{C}_{z}$ and $\hat{C}_{z}^{\prime \prime}$.

The precision results will be given for two cases, a zero-baseline and a short-baseline $(13 \mathrm{~km})$. The zerobaseline was measured as part of the 1995 ARI receiver test conducted by the University NAVSTAR Consortium (UNAVCO) [Rocken et al., 1995]. The experiment took place on Sept. $14^{\text {th }}, 1995$ around 23:30h (GPS) in Boulder, Colorado, US. The $13 \mathrm{~km}$ baseline was measured as part of a small network in the Flevopolder in the Netherlands on Dec. 22 ${ }^{\text {nd }}, 1996$, around 17:30h (GPS). 

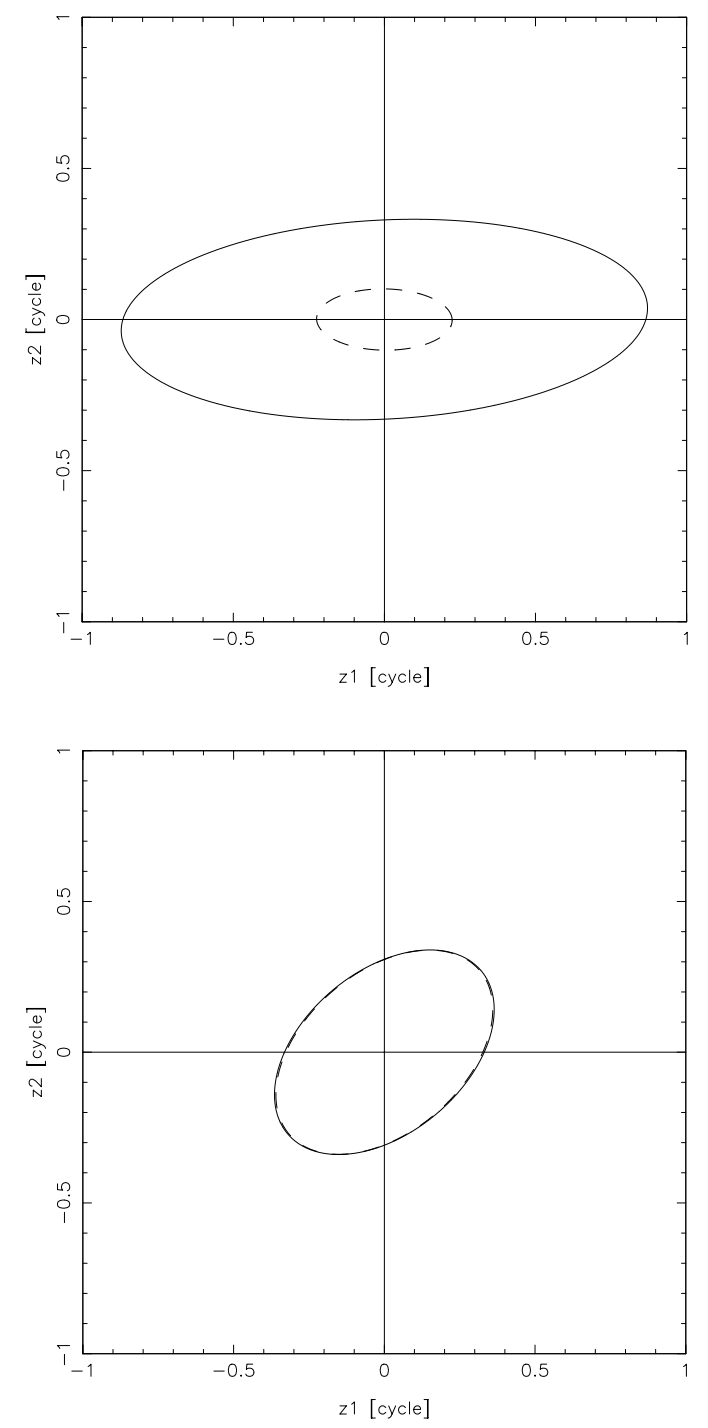

Figure 3: Formal and empirical standard ellipses of dual frequency zero-baseline ambiguity estimates; (top) $C_{z}$ (dashed) and $\hat{C}_{z}$ (full) of solution Eq.(4.2); (bottom) $C_{z}^{\prime \prime}$ (dashed) and $\hat{C}_{z}^{\prime \prime}$ (full) of solution Eq.(4.3).

Figure 3 shows typical standard ellipses for the zero baseline case. It can be seen from the figure that a significant disagreement exists between the standard ellipses corresponding to the formal and empirical precision of the ambiguities of Eq.(4.2). The formal standard ellipse appear to be far too optimistic. For the ambiguity estimates of Eq.(4.3) however, an almost perfect match is found for the formal and empirical standard ellipses. This illustrates that the nondiagonal matrices of Eq.(3.2) and Eq.(3.6) indeed give a more realistic description of the noise characteristics than simple diagonal matrices do.

For the short-baseline the results are less pronounced as a consequence of environmental effects in general and multipath effects in particular. Still the same trend can be seen. As figure 4 shows, the formal standard ellipse of Eq.(4.3) matches better with its empirical counterpart than the formal and empirical standard ellipses of Eq.(4.2) do.
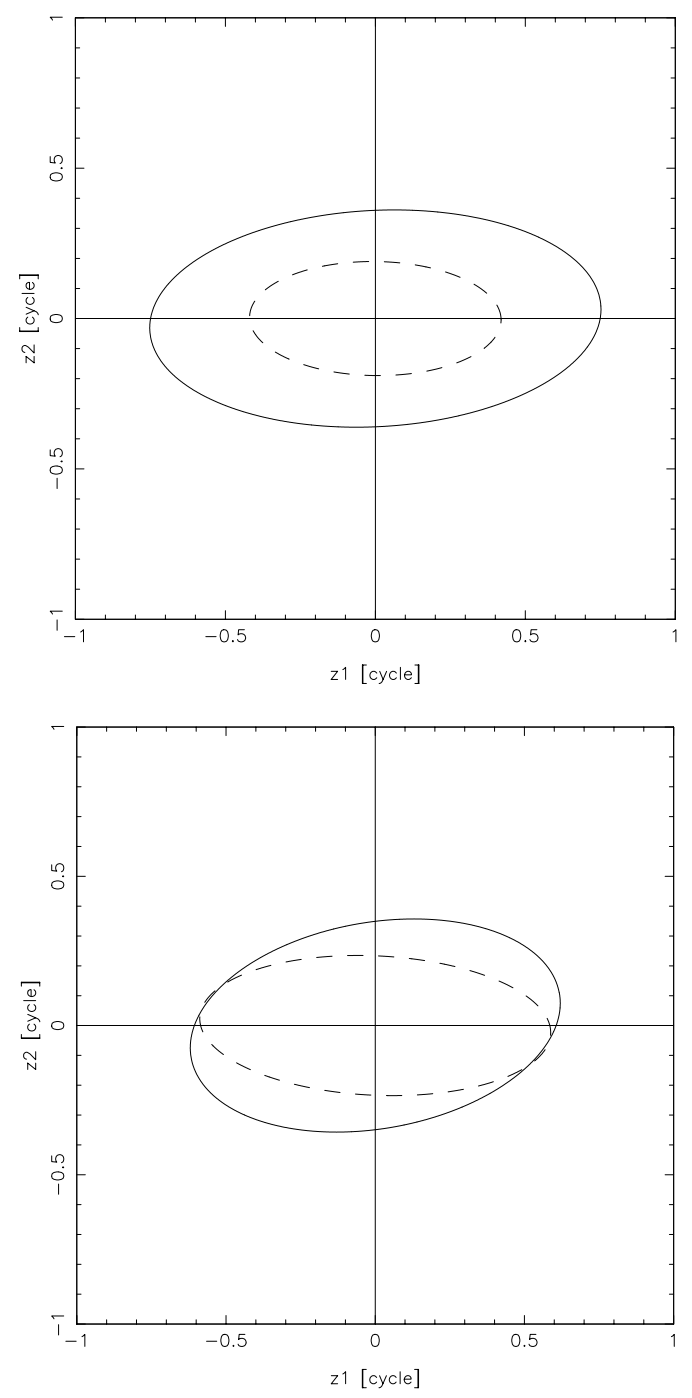

Figure 4: Formal and empirical standard ellipses of dual frequency short-baseline ambiguity estimates; (top) $C_{z}$ (dashed) and $\hat{C}_{z}$ (full) of solution Eq.(4.2); (bottom) $C_{z}^{\prime \prime}$ (dashed) and $\hat{C}_{z}^{\prime \prime}$ (full) of solution Eq.(4.3).

Apart from comparing the formal standard ellipse with its empirical counterpart, one can also compare the two empirical standard ellipses, the one of Eq.(4.2) with the one of Eq.(4.3). This comparison has the advantage that it is completely driven by the data themselves, since no formal variances and covariances need to be specified in order to compute the two solutions. Such a comparison is shown in figure 5 for three different cases. The first two are zero-baseline solutions, while the third is a short-baseline solution. 

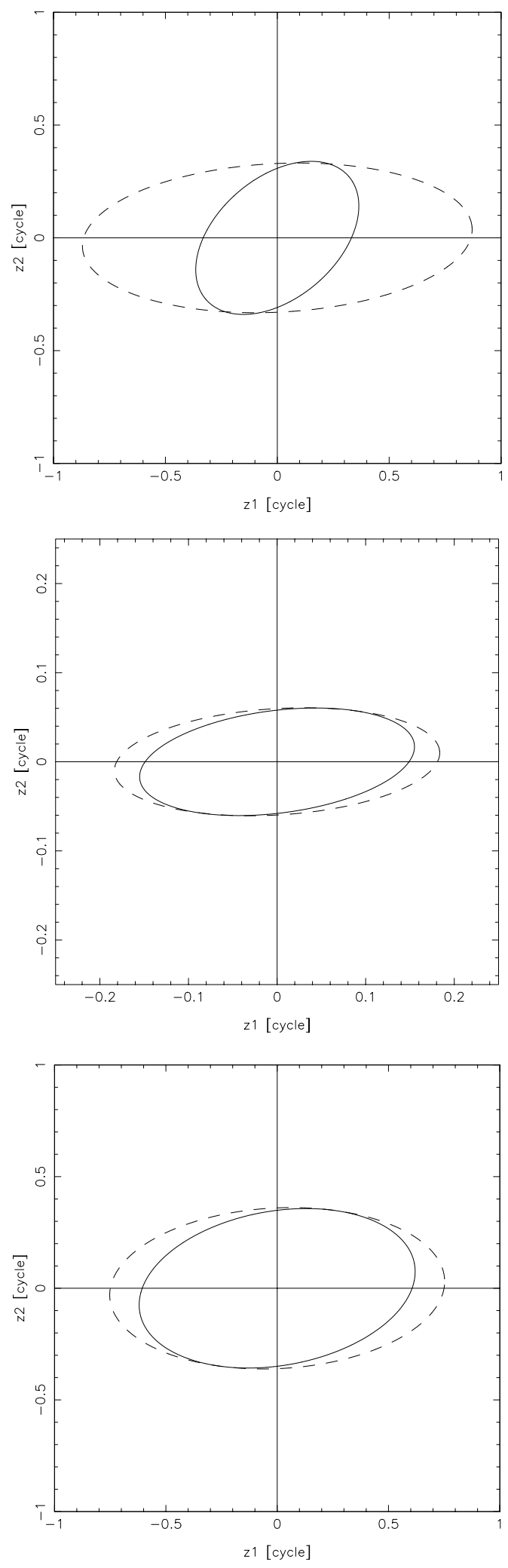

Figure 5: Comparison of the empirical ambiguity standard ellipses of Eq.(4.2) and Eq.(4.3); (top and middle) zero baseline, (bottom) short baseline; (all) $\hat{C}_{z}$ (dashed) and $\hat{C}_{z}^{\prime \prime}$ (full).

In all three cases, the empirical precision of Eq.(4.3) is better than that of Eq.(4.2). The difference is most pronounced in the first zero-baseline solution. This solution is based on DD data having one of its two satellites at a low elevation. The satellites of the other two solutions were at moderate elevations.

\section{DISCUSSION AND CONCLUSION}

By means of a simple analysis the correlation between the dual frequency observables of a cross-correlating receiver was revealed. The results presented appear to confirm that the nondiagonal vc-matrices $C_{p}$ and $C_{\phi}$ give a more realistic description of the noise characteristics of the data, than simple diagonal matrices would do. If that is the case, it has the following consequences for positioning with the surveyor's short-baseline model.

Let us first consider the pseudo range data. The dual frequency receiver outputs both $p_{1}$ and $p_{2}$. Would one use a diagonal vc-matrix for the pseudo range noise, one would assume the data to carry more information than it actually does. This is due to the fact that not $p_{1}$ and $p_{2}$ are the independent observables, but rather $p_{l}$ and $\Delta p$. For the latter pair a diagonal vc-matrix can be used, but not for the former. When using the appropriate vc-matrix, it automatically follows that no contribution can be expected from $p_{2}$. Only the L1 pseudo range contributes to the solution. This can be understood by noting that also $\Delta p$ fails to contribute, since in the short-baseline model, it has no model parameters in common with $p_{1}$. We thus end up in a remarkable situation: a more precise solution is obtained by not using one of the receiver-outputted observables, namely $p_{2}$. Hence we are better off by using less data. We may therefore conclude for this example, that although dual frequency data is made available by cross-correlation, it certainly does not imply the presence of a full GPS positioning capability when AntiSpoofing is turned on.

For the case of the carrier phase data the situation differs somewhat from the case of the pseudo range data. This is due to the fact that the carrier phase difference is measured in the cycle-domain and not in the range domain. Still a few remarks can be made. It is well-known that due to its longer wavelength, the widelane is often used for the purpose of ambiguity resolution, although this may even not be an argument in its own right [Teunissen, 1995]. It is therefore somewhat cynical to observe, that while the outputted L1 and L2 carrier phases are often transformed by the user to the widelane, it is actually this widelane which is the independent observable in the first place. With this transformation, the user will also obtain an erroneous widelane variance if he applies the error propagation, while assuming the L1 and L2 carrier phases to be independent. As a case in point, compare the widelane variance with the variance of the L2 carrier phase observable. In the context of ambiguity resolution, one often states as drawback of the widelane observable its higher noise level when compared to the noise level of the L1 and L2 carrier phases. This is certainly true when the L1 and L2 carrier phases are 
independent. But it is not necessarily true when the vcmatrix $C_{\phi}$ of Eq.(3.6) applies. In that case the variance of the L2 carrier could well be larger than the widelane variance, since it depends on how precise the widelane can be observed. These last remarks may in fact well explain the disagreement between the empirical and formal results presented in [Takac et al., 1998].

\section{REFERENCES}

Ashjaee, J., R. Lorenz (1992): Precision GPS surveying after Y-code. Proceedings of ION GPS-92, pp. 657659.

Barboux, J-P. (1997): Personal communication. Dassault Sercel NP, Carquefou, France.

Dierendonck, A.J. van (1995): Understanding GPS receiver technology: a tutorial. GPS World, pp. 3444.

Euler, H-J., C.C. Goad (1991): On optimal filtering of GPS dual frequency observations without using orbit information. Bulletin Géodésique, Vol. 65, pp. 130-143.

Gerdan, G.P. (1995): A comparison of four methods of weighting double difference pseudo range measurements. Trans Tasman Surveyor, Vol. 1, No. 1, pp. 60-66.

Gianniou, M. (1996): Genauigkeitssteigerung bei kurzzeit-statischen und kinematischen Satellitenmessungen bis hin zu Echtzeitanwendung. PhD thesis, Deutsche Geodätische Kommission, Reihe C, No. 458, München.

Gurtner, W. (1994): RINEX: Receiver Independent Exchange Format. GPS World, pp. 48-52.

Hofmann-Wellenhof, B., H. Lichtenegger, J. Collins (1997): GPS Theory and Practice. Fourth, revised edition, Springer Verlag, Wien/New York.

Jonkman, N.F. (1998): Integer GPS-ambiguity estimation without the receiver-satellite geometry. Publications of the Delft Geodetic Computing Centre, LGR Series, No. 18, 95 p.

Jin, X.X., C.D. de Jong (1996): Relationship between satellite elevation and precision of GPS code observations. The Journal of Navigation, Vol. 49, pp. 253-265.

Langley, R.B. (1996): GPS receivers and the observables. Chapter 4 in: GPS for Geodesy, A. Kleusberg and P.J.G. Teunissen (Eds.), Lecture Notes in Earth Sciences, Vol. 60, Springer Verlag.

MacDoran, P.F. (1979): Satellite emission radio interferometric earth surveying series - GPS geodetic system. Bulletin Géodésique, Vol. 53, pp. 117-138.

MacDoran, P.F., R.B. Miller, L. A. Buennagel, J.H. Whitcomb (1985): Codeless systems for positioning with NAVSTAR-GPS. Proceedings of the First International Symposium on Precise Positioning with the Global Positioning System, pp. 181-190.

Meehan, T.K., J.M. Srinivasan, D.J. Spitzmesser, C.E. Dunn, J.Y. Ten, J.B. Thomas, T.N. Munson, C.B. Duncan (1992): The TurboRogue GPS receiver.
Proceedings of the Sixth International Geodetic Symposium on Satellite Positioning, pp. 209-218.

Rocken, C., C. Meertens, B. Stephens, J. Braun, T. VanHove, S. Perry, O. Ruud, M. McCallum, J. Richardson (1995): UNAVCO Academic Research Infrastructure (ARI) receiver and antenna test report. Boulder, Colorado, US.

Takac, F., G.P. Gerdan, T.R. Lemmon, T.A. Hailes (1998). A new civilian GPS frequency: what can surveyors expect? The Australian Surveyor, Vol. 43, pp. 41-46.

Teunissen, P.J.G. (1995): The invertible GPS ambiguity transformations. Manuscripta Geodaetica, Vol. 20, pp. 489-497.

Tiberius, C.C.J.M. (1998): Recursive data processing for kinematic GPS surveying. Publications on Geodesy, Netherlands Geodetic Commission (in preparation).

Trimble Navigation Limited (1994): Trimble series 4000 receiver reference, Revision: A., 31 March, Sunnyvale, CA, US. 